\title{
Atenção Farmacêutica na Saúde Mental: Centro de Atenção Psicossocial
}

\author{
Pharmaceutical Care in Mental Health: Psychosocial Care Center \\ Atención farmacéutica en salud mental: Centro de atención psicosocial
}

Recebido: 09/09/2021 | Revisado: 20/09/2021 | Aceito: 05/10/2021 | Publicado: 09/10/2021

\author{
Cristiane Cervantes Ruiz \\ ORCID: https://orcid.org/0000-0002-1741-8660 \\ Faculdade Integrada Carajás, Brasil \\ E-mail: cristianecruiz@ hotmail.com \\ Milena Oliveira Queiroz \\ ORCID: https://orcid.org/0000-0002-5283-4256 \\ Faculdade Integrada Carajás, Brasil \\ E-mail: milesqueiroz@gmail.com \\ Yolanda de Jesus Morais \\ ORCID: https://orcid.org/0000-0001-7105-8267 \\ Faculdade Integrada Carajás, Brasil \\ E-mail: farmaceuticayolandamorais5004@gmail.com
}

\begin{abstract}
Resumo
Objetivo: Estudar a atuação do farmacêutico na saúde mental no CAPS, pois, nesse centro o farmacêutico é essencial na atuação com pacientes que têm transtorno mental e a utilização racional dos medicamentos deve passar pela assistência farmacêutica. Métodos: Revisão integrativa com síntese narrativa. Resultados: Foram discutidos 15 estudos de modo a fundamentar esta revisão integrativa e a partir deles concluiu-se que a atenção farmacêutica é fundamental tanto na orientação dos pacientes, familiares, quanto a própria equipe CAPS, isso porque na falta de medicamentos referência é possível a substituição por medicamentos genéricos sem prejudicar o tratamento do paciente. Conclusão: O farmacêutico é um profissional fundamental porque ele detém o conhecimento técnico dos medicamentos, e há uma necessidade de informação aos pacientes e familiares no campo da saúde mental, através deste profissional o atendimento torna-se integral tanto no que se refere às ações técnico-gerenciais quanto às ações técnico-assistenciais exercidas dentro do CAPS.
\end{abstract}

Palavras-chave: Fármacos; Medicamentos Antipsicóticos; Psicotrópicos; Assistência; Orientação.

\begin{abstract}
Objective: to study the role of the pharmacist in mental health at CAPS, as the pharmacist at this center is essential in working with patients who have mental disorders and the rational use of medications must include pharmaceutical care. Methods: Integrative review with narrative synthesis. Results: 15 studies were discussed in order to support this integrative review and from them it was concluded that pharmaceutical care is essential both in the guidance of patients, family members, the CAPS team itself, because in the absence of reference drugs, a replacement is possible. for generic drugs without harming the patient's treatment. Conclusion: The pharmacist is a fundamental professional because he has the technical knowledge of medicines, and there is a need for information to patients and families in the field of mental health, through this professional the technical service becomes integral both with regard to actions -managerial regarding the technical-assistance actions carried out within the CAPS.
\end{abstract}

Keywords: Drugs; Antipsychotic Medicines; Psychotropics; Assistance; Guidance.

\section{Resumen}

Objetivo: estudiar el papel del farmacéutico en la salud mental en el CAPS, ya que en este centro el farmacéutico es fundamental para trabajar con pacientes con trastornos mentales y el uso racional de los medicamentos debe incluir la atención farmacéutica. Métodos: Revisión integrativa con síntesis narrativa. Resultados: se discutieron 15 estudios para sustentar esta revisión integradora y de ellos se concluyó que la atención farmacéutica es fundamental tanto en la orientación de los pacientes, familiares, del propio equipo CAPS, pues en ausencia de medicamentos de referencia se hace un reemplazo. posible. para medicamentos genéricos sin dañar el tratamiento del paciente. Conclusión: El farmacéutico es un profesional fundamental porque posee el conocimiento técnico de los medicamentos, y existe una necesidad de información a los pacientes y familiares en el campo de la salud mental, a través de este profesional el servicio técnico se vuelve integral tanto en lo que se refiere a las acciones -gestivas en cuanto a las acciones de asistencia técnica realizadas dentro de los CAPS.

Palabras clave: Drogas; Medicamentos Antipsicóticos; Psicotrópicos; Asistencia; Guia. 


\section{Introdução}

A atenção farmacêutica passou por um processo histórico de construção porque com o decorrer do tempo o paciente com problemas de saúde de mental passou a valorizado e os tratamentos medicamentosos deixaram de apresentar características de exclusão social e começaram a tratar sobre formas de controle dos sintomas, favorecendo assim a humanização do trabalho farmacêutico.

\section{Processo histórico da doença mental}

Por muitos anos, pessoas com diagnóstico de doença mental foram tratadas em instituições cujo princípio básico de tratamento é o isolamento. O objetivo era manter os "lunáticos" longe da vida social. Pessoas pertencentes a famílias ricas recebiam um tratamento diferente, eles ermam presos em suas casas ou sanatórios, enquanto os pobres estavam nas ruas ou abrigados em Santas Casas de Misericórdia (Coutinho, 2015).

As regras rígidas e as excessivas medidas de segurança contribuíam significativamente para piora do quadro clínico destes pacientes, além da falta de fármacos adequados e ainda havia um reforço para concepção social que estes pacientes representavam um perigo para sociedade, reafirmando a necessidade de excluí-los e ainda, promover um distanciamento de suas famílias (Santos, 2018).

Apenas no início do século XX é que surge a psicofarmacologia e com ela fármacos voltados ao tratamento e controle das questões pertinentes a saúde mental dos pacientes, em 1980 surge uma mobilização política e social em prol do processo de redemocratização no Brasil, que foi determinante para o movimento da reforma sanitária e também contribuiu para uma nova forma de pensar a saúde mental (Silva; Lima, 2017).

\section{Evolução nacional através da Constituição Federal e legislações}

A Constituição Federal representou um marco na garantia de direitos e deveres de toda a sociedade e partir dela formulou-se uma legislação que visa padronizar e melhorar o atendimento aos pacientes com transtornos mentais nos diferentes níveis de atenção à saúde. Desde então, o campo da saúde vem se reconfigurando com novos saberes e novas práticas, o que faz com que os profissionais mudem suas percepções sobre os transtornos mentais e o tratamento, e reflitam sobre o cuidado prestado a esses pacientes (Silva; Lima, 2017).

Durante a implantação da reforma sanitária e do Sistema Único de Saúde (SUS), no início da década de 1990, o Brasil também aplicou novos tratamentos na área de saúde mental, e posteriormente oportunizou a criação de um Centro de Atenção Psicossocial (CAPS), seu principal objetivo é permitir que o paciente se reintegre à sociedade e evite ser rejeitado por internação psiquiátrica desnecessária (Brasil, 2002b).

A partir da Lei nº 10.216 de 4 de junho de 2001 (Brasil, 2001a), houve a incorporação de melhorias nos serviços e assistência às pessoas com transtornos mentais, enfatizando mais humanidade e respeito aos direitos dos pacientes. Nesse caso, o Centro de Atenção Psicossocial (CAPS) foi incorporado à política de saúde mental por meio da Portaria $\mathrm{n}^{\circ} 336$, de 19 de fevereiro de 2002 (Brasil, 2002a), que dispõe sobre as normas e diretrizes para a prestação de serviços de saúde mental.

\section{Papel da reforma psiquiátrica na assistência farmacêutica}

Nesse sentido, destacou-se ao longo do trabalho o papel da reforma psiquiátrica brasileira e seu impacto no trabalho exercido na assistência farmacêutica, ou seja, uma série de ações e serviços para garantir um tratamento e assistência integral, promoção e reabilitação da saúde, incluindo atividades de pesquisa, seleção, planejamento, acesso, armazenamento, distribuição, dispensação e promoção do uso racional de medicamentos. São entendidos como insumos básicos de saúde que devem ser acessíveis e razoavelmente utilizados (Brasil, 2004a). 
Nesses conjuntos de ações, o CAPS deve ser referência para distribuição de medicamentos para saúde mental. Porém, sabe-se que as estratégias de saúde da família também são responsáveis nesse processo, devendo haver uma equipe que dê assistência suficiente para que esse grupo de usuários atenda às diferentes necessidades e objetivos de saúde mental e saúde. Política de assistência medicamentosa, em especial a assistência relacionada ao fornecimento, distribuição e promoção do uso racional de medicamentos.

Nesse sentido, ainda persistem lacunas no processo de reposicionamento da assistência farmacêutica, o que rompe a lógica das atividades e afeta diretamente a saúde dos portadores de transtorno mental. Diante dessa realidade, é necessário reexaminar os serviços que vêm sendo desenvolvidos para garantir a esses usuários uma melhor assistência farmacêutica, pois o uso racional de medicamentos é essencial para o tratamento dos transtornos mentais.

Diante do que já foi citado, o objetivo deste trabalho é estudar a atuação do farmacêutico na saúde mental no CAPS, pois, nesse centro o farmacêutico é essencial na atuação com pacientes que têm transtorno mental e a utilização racional dos medicamentos deve passar pela assistência farmacêutica.

\section{Método}

Trata-se de uma revisão integrativa (Cunha; Cunha; Alves, 2014), apresentado como questão norteadora a necessidade da atenção farmacêutica dentro do contexto do CAPS, pois é um centro de atenção público direcionado ao atendimento de pacientes com problemas de saúde mentais e que fornece aqueles que não têm condições de pagar por um tratamento uma atenção completa de diversos profissionais, e o farmacêutico deve exercer uma função informativa, proativa e de gestão. O recorte temporal ocorre entre os anos de 2013 a 2021, utilizando as seguintes plataformas de dados científicos: Scielo Brasil, Google Acadêmico e Lilacs. A estratégia de busca apresentada por Cunha, Cunha e Alves (2014) em que identifica-se o tema e seleciona-se a questão de pesquisa para selecionar os artigos a serem utilizados. Restringiram-se a pesquisa as línguas portuguesa, espanhola e inglesa.

Utilizaram-se como critérios de inclusão: Temática; Temporalidade; Apresentação de Resultados; Explicações sobre a atuação farmacêutica no CAPS; Desfecho: melhora na atenção farmacêutica; orientação clara dos efeitos medicamentosos. Tipos de estudos: Revisão bibliográfica, sistemática, integrativa, relato de experiência, estudo transversal e foram excluídos os manuscritos repetidos ou duplicados fora do período definido para o estudo e sem adequação aos objetivos da pesquisa.

Identificaram-se no total 259 publicações, sendo estes: 200 no Google Escolar, 9 no SciELO, 50 no Lilacs, após aplicação dos critérios de inclusão, foram excluídos 244 estudos identificados de modo que através da análise de pertinência à temática restaram 15 estudos analisados a seguir. Os resultados apresentados acima foram dispostos ao Fluxograma 1. 
Fluxograma 1: Fluxograma com as etapas de inclusão dos artigos.
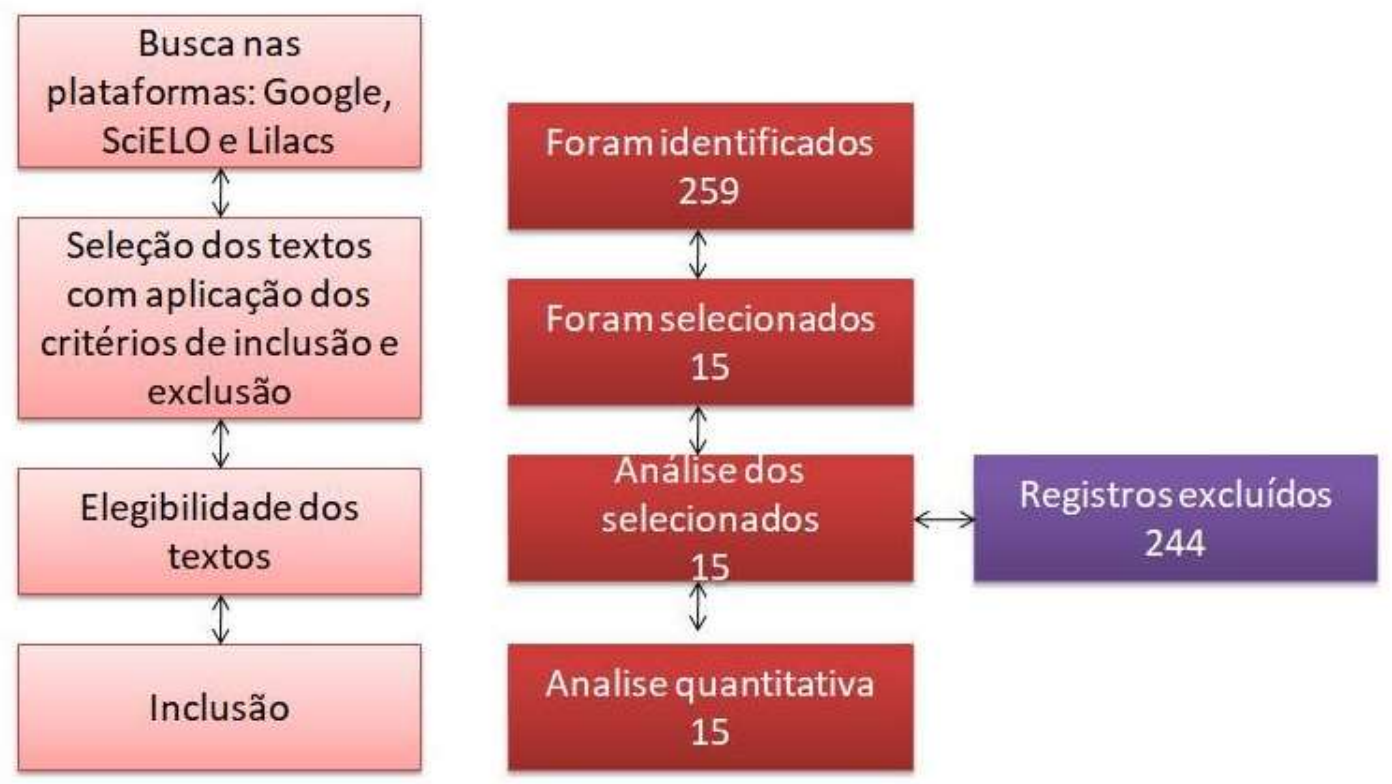

Fonte: Atenção Farmacêutica na Saúde Mental: Centro de Atenção Psicossocial (2021).

\section{Resultados e Discussão}

Foram incluídos 15 estudos para integrar este artigo de revisão integrativa, sendo 7 estudos transversais, 1 revisão bibliográfica, 3 estudos observacionais transversais, 2 revisões integrativas, 2 revisões de literatura, os autores do artigos, objetivos, a metodologia utilizada no estudo e o desfecho.

Os artigos analisados apresentam os seguintes critérios, o uso racional do medicamento, competência de gestão, assistência à família, assistência ao paciente e assistência no CAPS, conforme demonstrado na tabela 2.

Alencar (2013), Bezerra (2013), Zanella; Aguiar; Storpirtis (2015), Carvalho et al., (2015), Almeida et al., (2016), Silva; Lima (2017), Beutinger; Limberger (2019) concluem em seus estudos transversais que a presença do farmacêutico no CAPS é importante para manter o uso racional dos medicamentos através da conscientização do paciente e análise dos medicamentos que já estejam em uso.

Correia; Gondim (2014) em suas revisões bibliográficas ressaltaram que o acompanhamento farmacêutico é importante para compreender as nuances da saúde mental.

Silva (2014), Smanio et al., (2018), Guimarães (2020), em seus estudos observacionais transversais foram constatadas as necessidades de mudanças estruturais e de processos de trabalho, como capacitação dos funcionários das farmácias/dispensários de saúde.

Leite et al., (2016), Amaral et al., (2021) nas revisões integrativas destacaram a importância da gestão farmacêutica, bem como a assistência prestada à família, usuário e equipe.

Scherer Berwing; Silva (2017), Santos (2018), nas revisões de literatura apresentaram os resultados pertinentes à assistência farmacêutica a família e usuários, e da necessidade de investimento em equipamentos e conhecimento. 
Tabela 1: Publicações que apresentam a interação entre antibióticos e anticoncepcionais no organismo feminino.

\begin{tabular}{|c|c|c|c|}
\hline ARTIGO & OBJETIVO & $\begin{array}{l}\text { METODOLOGIA } \\
\text { DO ESTUDO }\end{array}$ & DESFECHO \\
\hline Alencar (2013) & $\begin{array}{l}\text { Analisar o processo de trabalho } \\
\text { em saúde, no que concerne às } \\
\text { ações relacionadas à } \\
\text { assistência farmacêutica no } \\
\text { CAPS. }\end{array}$ & Estudo transversal. & $\begin{array}{l}\text { A assistência farmacêutica é fundamental para } \\
\text { integração das ações gerenciais concernentes a } \\
\text { seleção, programação, aquisição, armazenamento e } \\
\text { distribuição além de contribuir para o uso racional } \\
\text { de medicamentos. }\end{array}$ \\
\hline Bezerra (2013) & $\begin{array}{l}\text { Discutir a gestão do uso de } \\
\text { medicamentos por usuário do } \\
\text { CAPS e como o uso de } \\
\text { psicofármacos r resulta } \\
\text { positivamente no quadro dos } \\
\text { pacientes. }\end{array}$ & Estudo transversal. & $\begin{array}{l}\text { Concluiu-se que o CAPS deve operacionalizar as } \\
\text { ações de modo que todas as etapas resultem em um } \\
\text { usuário informado e participativo, e que as etapas de } \\
\text { atendimento, relação com equipe e ministração } \\
\text { medicamentosa deve abranger a assistência } \\
\text { farmacêutica, por ser este o profissional que detém o } \\
\text { conhecimento dos fármacos. }\end{array}$ \\
\hline $\begin{array}{l}\text { Correia; } \quad \text { Gondim } \\
\text { (2014) }\end{array}$ & $\begin{array}{l}\text { Realizar revisão bibliográfica } \\
\text { sobre saúde mental e o uso de } \\
\text { benzodiazepínicos por } \\
\text { pacientes portadores de algum } \\
\text { transtorno mental. }\end{array}$ & $\begin{array}{c}\text { Revisão } \\
\text { Bibliográfica. }\end{array}$ & $\begin{array}{l}\text { O acompanhamento fármaco terapêutico requer além } \\
\text { da ministração medicamentosa a orientação e } \\
\text { convencimento do paciente que possui transtornos } \\
\text { mentais sobre o beneficio da medicação, isso porque } \\
\text { ainda, que haja um familiar ou um profissional } \\
\text { acompanhando o paciente se este não quiser fazer } \\
\text { uso do medicamento não se pode obrigar o paciente, } \\
\text { é nesse sentido que o acompanhamento farmacêutico } \\
\text { é importante para compreender as nuances da saúde } \\
\text { mental. }\end{array}$ \\
\hline Silva (2014) & $\begin{array}{l}\text { Analisar a estrutura no CAPS e } \\
\text { a organização na Assistência } \\
\text { Farmacêutica nestes serviços. }\end{array}$ & $\begin{array}{l}\text { Estudo observacional } \\
\text { transversal. }\end{array}$ & $\begin{array}{l}\text { Ao realizar o estudo observacional percebeu-se a } \\
\text { limitação da assistência farmacêutica no CAPS, } \\
\text { pois, o farmacêutico não era incluído efetivamente } \\
\text { nas atividades do Centro e destacou que o } \\
\text { farmacêutico não deve ser um profissional que } \\
\text { cumpra apenas a obrigatoriedade de uma norma } \\
\text { sanitária por estar presente, mas, que seja } \\
\text { participativo. }\end{array}$ \\
\hline $\begin{array}{l}\text { Zanella; Aguiar; } \\
\text { Storpirtis (2015) }\end{array}$ & $\begin{array}{l}\text { Avaliar a atuação do } \\
\text { farmacêutico na dispensação } \\
\text { de medicamentos, sendo } \\
\text { realizada pesquisa transversal } \\
\text { exploratório-descritiva em oito } \\
\text { Centros de Atenção } \\
\text { Psicossocial (CAPS) Adulto do } \\
\text { Município de São Paulo. }\end{array}$ & $\begin{array}{c}\text { Estudo } \\
\text { Transversal. }\end{array}$ & $\begin{array}{l}\text { As práticas atuais de atendimento ao paciente estão } \\
\text { sendo desenvolvidas nas unidades de CAPS, que } \\
\text { possuem a presença de um farmacêutico. Na amostra } \\
\text { da pesquisa, observou-se que poucos profissionais } \\
\text { realizaram todas as prescrições de distribuição e } \\
\text { avaliação. No passado, porque os farmacêuticos não } \\
\text { costumavam discutir medicamentos com os } \\
\text { médicos. }\end{array}$ \\
\hline $\begin{array}{l}\text { Carvalho et al., } \\
(2015)\end{array}$ & $\begin{array}{l}\text { Analisar o perfil de tratamento } \\
\text { medicamentoso dos } \\
\text { adolescentes nos CAPS - } \\
\text { Infanto-Juvenil do Município } \\
\text { de Fortaleza - CE. }\end{array}$ & $\begin{array}{c}\text { Estudo } \\
\text { Transversal }\end{array}$ & $\begin{array}{l}\text { Nesta pesquisa ao longo da pesquisa foi identificada } \\
\text { a utilização dos seguintes psicotrópicos } \\
\text { antipsicóticos e neurolépticos representando } 37,5 \% \text {, } \\
\text { seguido pelos antidepressivos representado } 22,2 \% \text {. } \\
\text { Foi constatado também que os jovens reconhecem os } \\
\text { medicamentos pelo nome. }\end{array}$ \\
\hline Leite et al., (2016) & $\begin{array}{l}\text { Analisar a importância da } \\
\text { atenção farmacêutica, sendo } \\
\text { identificadas a incidência de } \\
\text { reações adversas e interações } \\
\text { medicamentosas, bem como o } \\
\text { papel do farmacêutico no } \\
\text { CAPS nesse sentido. }\end{array}$ & $\begin{array}{c}\text { Revisão } \\
\text { Integrativa. }\end{array}$ & $\begin{array}{l}\text { Por se compreender que os transtornos psiquiátricos } \\
\text { exigem a utilização de medicamentos psicotrópicos } \\
\text { o farmacêutico é importante para realizar a análise } \\
\text { das prescrições, orientação dos usuários, } \\
\text { dispensação e gestão dos medicamentos, assim no } \\
\text { CAPS o farmacêutico exerce múltiplas funções. }\end{array}$ \\
\hline Almeida et al., (2016) & $\begin{array}{l}\text { Analisar a aplicação do passo } 2 \\
\text { do GGAM em um grupo de } \\
\text { cuidadores de } \quad \text { adolescentes }\end{array}$ & $\begin{array}{c}\text { Estudo } \\
\text { transversal. }\end{array}$ & $\begin{array}{l}\text { Pode-se concluir que existe apenas um contato entre } \\
\text { profissionais de saúde e cuidadores de adolescentes. } \\
\text { Nesse sentido, o Gestão Autônoma da Medicação }\end{array}$ \\
\hline
\end{tabular}




\begin{tabular}{|c|c|c|c|}
\hline & $\begin{array}{l}\text { com deficiência intelectual em } \\
\text { um Centro de Atenção } \\
\text { Psicossocial Infantojuvenil }\end{array}$ & & $\begin{array}{l}\text { GGAM mostra que cuidadores que encaram o } \\
\text { conhecimento podem contribuir para o melhor } \\
\text { desenvolvimento de sua autonomia no cuidado ao } \\
\text { adolescente. }\end{array}$ \\
\hline Silva; Lima (2017) & $\begin{array}{l}\text { Avaliar a assistência } \\
\text { farmacêutica nos CAPS por } \\
\text { meio de um estudo transversal } \\
\text { em 15 CAPS. }\end{array}$ & $\begin{array}{l}\text { Estudo } \\
\text { transversal. }\end{array}$ & $\begin{array}{l}\text { O farmacêutico foi identificado dentro do CAPS } \\
\text { exercendo o controle e gestão dos medicamentos na } \\
\text { responsabilidade das farmácias, bem como nas } \\
\text { atividades de assistência ao paciente de modo que } \\
\text { estes compreendam o que e o por que estão fazendo } \\
\text { uso dos medicamentos psicotrópicos. }\end{array}$ \\
\hline $\begin{array}{l}\text { Scherer Berwing; } \\
\text { Silva (2017) }\end{array}$ & $\begin{array}{l}\text { Comprovar e colocar em } \\
\text { evidência as contribuições do } \\
\text { profissional farmacêutico no } \\
\text { CAPS. }\end{array}$ & Revisão de literatura. & $\begin{array}{l}\text { Acredita-se que uma das maiores fragilidades do } \\
\text { sistema seja o foco das ações de saúde na } \\
\text { acessibilidade dos medicamentos, e em muitos casos } \\
\text { não tem a ver com o uso racional dos medicamentos. } \\
\text { o farmacêutico no CAPS pode integrar uma equipe } \\
\text { interdisciplinar de saúde centrada no usuário para } \\
\text { promover, proteger, restaurar a saúde e prevenir } \\
\text { agravos, dormir à educação em saúde e promover o } \\
\text { uso de medicamentos e medicamentos prescritos } \\
\text { racional. }\end{array}$ \\
\hline Santos (2018) & $\begin{array}{l}\text { Analisar a contribuição do } \\
\text { farmacêutico na qualidade de } \\
\text { vida de pacientes com } \\
\text { transtornos mentais no CAPS. }\end{array}$ & Revisão de literatura. & $\begin{array}{l}\text { Concluiu-se que o farmacêutico é importante para } \\
\text { utilização adequada dos psicotrópicos } \\
\text { principalmente na prevenção de dependência } \\
\text { medicamentosa, e que a compreensão do paciente ao } \\
\text { tratamento farmacológico contribui para melhoria na } \\
\text { qualidade vida e evita automedicação. }\end{array}$ \\
\hline Smanio et al., (2018) & $\begin{array}{l}\text { Relatar as ações técnico- } \\
\text { gerenciais e assistenciais da } \\
\text { assistência farmacêutica de um } \\
\text { Centro de Atenção } \\
\text { Psicossocial e Droga a partir } \\
\text { da contratação do farmacêutico } \\
\text { e o impacto dessas atividades } \\
\text { na melhoria da qualidade de } \\
\text { assistência e do cuidado com } \\
\text { paciente. }\end{array}$ & $\begin{array}{l}\text { Estudo observacional } \\
\text { transversal. }\end{array}$ & $\begin{array}{l}\text { A estrutura física do CAPS, o auxílio de toda equipe } \\
\text { e a presença de um farmacêutico são benéficas ao } \\
\text { usuário, porque ambos os aspectos contribuem para } \\
\text { organização, conhecimento e gestão dos recursos } \\
\text { necessários para manutenção de um tratamento. }\end{array}$ \\
\hline Santos (2019) & $\begin{array}{l}\text { Investigar a } \text { necessidade da } \\
\text { implantação de } \quad \text { serviços } \\
\text { farmacêticos } \\
\text { acompanhamento de jovens } \\
\text { portadores de transtornos } \\
\text { mentais e usuários de fármacos } \\
\text { psicoativos no CAPS. }\end{array}$ & $\begin{array}{l}\text { Estudo observacional } \\
\text { transversal. }\end{array}$ & $\begin{array}{l}\text { Por meio dos serviços de assistência farmacêutica } \\
\text { houve a melhor qualidade de atendimento durante o } \\
\text { tratamento, pois a farmacêutica conta com todo o } \\
\text { seu conhecimento em Farmacologia, atuando em } \\
\text { conjunto com outros profissionais para auxiliar os } \\
\text { pacientes no planejamento e acompanhamento de } \\
\text { planos terapêuticos específicos e adequados. }\end{array}$ \\
\hline $\begin{array}{l}\text { Beutinger; Limberger } \\
\text { (2019) }\end{array}$ & $\begin{array}{l}\text { Proporcionar um atendimento } \\
\text { integral e humanizado aos } \\
\text { usuários através do Projeto } \\
\text { Terapêutico Singular. }\end{array}$ & $\begin{array}{c}\text { Estudo } \\
\text { transversal. }\end{array}$ & $\begin{array}{l}\text { O papel do farmacêutico é fundamental, pois ele se } \\
\text { dedica aos serviços de farmácia, promovendo a } \\
\text { acessibilidade e fornecendo orientações racionais } \\
\text { sobre medicamentos. Obviamente, o papel do } \\
\text { farmacêutico junto à equipe e ao usuário traz muitos } \\
\text { benefícios clínicos e econômicos, pois resolve e } \\
\text { previne problemas relacionados à medicação. Os } \\
\text { usuários de saúde mental são imprescindíveis porque } \\
\text { os farmacêuticos ajudam a solucionar as } \\
\text { dificuldades enfrentadas pelo público em lidar com } \\
\text { os tratamentos e fornecem-lhes mais conhecimentos } \\
\text { sobre o tratamento, para que a qualidade de vida dos } \\
\text { sujeitos seja restaurada ou mantida. }\end{array}$ \\
\hline $\begin{array}{l}\text { Guimarães } \\
\text { (2020) }\end{array}$ & $\begin{array}{l}\text { Avaliar a assistência } \\
\text { farmacêutica no contexto da } \\
\text { Atenção primária à saúde } \\
\text { (APS) em um município da }\end{array}$ & $\begin{array}{l}\text { Estudo observacional } \\
\text { transversal. }\end{array}$ & $\begin{array}{l}\text { Com esse estudo, foi constatada a necessidade de } \\
\text { mudanças estruturais e de processos de trabalho, } \\
\text { como capacitação dos funcionários das } \\
\text { farmácias/dispensários de saúde. Outra necessidade }\end{array}$ \\
\hline
\end{tabular}




\begin{tabular}{|c|c|c|c|}
\hline & região nordeste do Brasil. & & $\begin{array}{l}\text { seria a inclusão de profissionais especializados para } \\
\text { qualificar e ampliar as ações da assistência } \\
\text { farmacêutica na APS, como a implantação de } \\
\text { registros para movimentação de estoque e } \\
\text { identificação das demandas reprimidas para reportar } \\
\text { para a gestão, o aumento da disponibilidade dos } \\
\text { medicamentos chaves, a implantação de serviços de } \\
\text { cuidado farmacêutico para usuários que possuem } \\
\text { dificuldade de adesão ao tratamento. }\end{array}$ \\
\hline Amaral et al., (2021) & $\begin{array}{l}\text { Avaliar as características das } \\
\text { redes de saúde de quatro } \\
\text { grandes municípios brasileiros } \\
\text { (Campinas, Fortaleza, Porto } \\
\text { Alegre e São Paulo) no que diz } \\
\text { respeito à prestação de } \\
\text { cuidados em saúde mental. }\end{array}$ & $\begin{array}{c}\text { Revisão } \\
\text { Integrativa. }\end{array}$ & $\begin{array}{l}\text { A prestação de serviços farmacêuticos a usuários } \\
\text { com transtornos mentais também é considerada um } \\
\text { desafio para a rede de saúde estudada. Vale ressaltar } \\
\text { que a prescrição de psicofármacos da AB ainda é } \\
\text { baixa, principalmente nas cidades de Campinas e } \\
\text { Fortaleza. Os resultados nesta dimensão apontaram } \\
\text { também para o problema da continuidade do } \\
\text { tratamento medicamentoso, particularmente } \\
\text { desvantajoso em Fortaleza e Porto Alegre devido à } \\
\text { falta de medicamentos prescritos nas farmácias } \\
\text { utilizadas pelos usuários. }\end{array}$ \\
\hline
\end{tabular}

Fonte: Atenção Farmacêutica na Saúde Mental: Centro de Atenção Psicossocial (2021).

Tabela 2: Desfechos apresentados sobre a atuação do farmacêutico no CAPS.

\begin{tabular}{|c|c|c|c|c|c|}
\hline ARTIGO & $\begin{array}{l}\text { Uso racional do } \\
\text { medicamento }\end{array}$ & $\begin{array}{l}\text { Competência de } \\
\text { Gestão }\end{array}$ & $\begin{array}{l}\text { Assistência à } \\
\text { família }\end{array}$ & $\begin{array}{c}\text { Assistência ao } \\
\text { paciente }\end{array}$ & $\begin{array}{c}\text { Assistência no } \\
\text { CAPS }\end{array}$ \\
\hline Alencar (2013) & $(+)$ & & $(+)$ & $(+)$ & $(+)$ \\
\hline Bezerra (2013) & $(+)$ & $(+)$ & $(+)$ & $(+)$ & $(+)$ \\
\hline $\begin{array}{c}\text { Correia; } \\
\text { Gondim (2014) }\end{array}$ & $(+)$ & & & $(+)$ & $(+)$ \\
\hline Silva (2014) & & $(+)$ & & & $(+)$ \\
\hline $\begin{array}{l}\text { Zanella; Aguiar; } \\
\text { Storpirtis (2015) }\end{array}$ & $(+)$ & $(+)$ & & $(+)$ & $(+)$ \\
\hline $\begin{array}{l}\text { Carvalho et al., } \\
\text { (2015) }\end{array}$ & $(+)$ & $(+)$ & & $(+)$ & $(+)$ \\
\hline $\begin{array}{l}\text { Leite } \text { et al., } \\
\text { (2016) }\end{array}$ & $(+)$ & $(+)$ & $(+)$ & $(+)$ & $(+)$ \\
\hline Almeida (2016) & $(+)$ & $(+)$ & $(+)$ & $(+)$ & $(+)$ \\
\hline $\begin{array}{l}\text { Silva; Lima } \\
\text { (2017) }\end{array}$ & $(+)$ & $(+)$ & & & $(+)$ \\
\hline $\begin{array}{c}\text { Scherer } \\
\text { Berwing; } \\
\text { Santiago (2017) }\end{array}$ & $(+)$ & $(+)$ & $(+)$ & & \\
\hline Santos (2018) & $(+)$ & & $(+)$ & $(+)$ & $(+)$ \\
\hline $\begin{array}{c}\text { Smanio et al., } \\
\quad(2018)\end{array}$ & $(+)$ & $(+)$ & & & $(+)$ \\
\hline Santos (2019) & $(+)$ & & $(+)$ & $(+)$ & $(+)$ \\
\hline
\end{tabular}




\begin{tabular}{c|c|c|c|c|c}
\hline $\begin{array}{c}\text { Beutinger; } \\
\text { Limberger } \\
(2019)\end{array}$ & $(+)$ & & $(+)$ & $(+)$ & $(+)$ \\
\hline $\begin{array}{c}\text { Guimarães } \\
(2020)\end{array}$ & $(+)$ & $(+)$ & $(+)$ & & \\
\hline $\begin{array}{c}\text { Amaral } \text { et al., } \\
(2021)\end{array}$ & $(+)$ & $(+)$ & $(+)$ & & \\
\hline
\end{tabular}

Fonte: Atenção Farmacêutica na Saúde Mental: Centro de Atenção Psicossocial (2021).

Alencar (2013) identificou que os profissionais possuem uma dificuldade de compreender seu papel dentro de instituições em que se torna necessário um trabalho interdisciplinar, isso porque apesar de cada profissional exercer uma especificidade a nomeclatura do ambiente tende a influenciar a compreensão geral do trabalho ali realizado, neste estudo o foco foi a atuação profissional do farmacêutico no Centro de Atenção Psicossocial, ou seja, os profissionais tendem a acreditar que o principal trabalho ali realizado é o psicológico através da figura do médico, assim através da aplicação do questionários e análise destes, percebeu-se que os farmacêuticos no CAPS são responsáveis por atividades educativas e que promovam a utilização racional dos medicamentos nas unidades e ainda que os pacientes tenham clareza sobre o princípio ativo medicamentoso que estará sendo ingerido.

Bezerra (2013) discutiu o processo de gestão e de uso dos medicamentos no CAPS e como a relação entre a família, equipe e paciente é fundamental para o sucesso do tratamento e controle dos sintomas da doença mental, e que através do farmacêutico foi possível compreender que existe a possibilidade de desenvolvimento da autonomia e responsabilização do paciente de modo que este compreenda quais medicamentos está em uso e por que está em uso deles.

Correia e Gondim (2014) identificaram que os benzodiazepínicos são fundamentais para o tratamento de doenças de cunho mental, tratadas nos CAPS e nas UBS - Unidades de Saúde Básica da Família e que através do enfoque farmacêutico esse tratamento torna-se claro ao paciente, que via de regra, possui uma resistência a esta por acreditar que o remédio poderá "apagá-lo", "fazer dormir demais", ou até mesmo "morrer", o conhecimento sobre o princípio ativo medicamentoso é fundamental e o farmacêutico passa a exercer uma prática assistencial ao oferecer aos pacientes informações sobre os medicamentos, possíveis efeitos colaterais de modo que o uso racional seja disseminado.

Silva (2014) através deste estudo demonstrou-se que o farmacêutico deve ser incluído nas atividades do CAPS e fazer parte da equipe multidisciplinar atuante do centro, tendo em vista que este profissional não pode apenas entregar medicamentos sem aplicar seu conhecimento e promover a assistência farmacêutica que é benéfica aos pacientes, além das questões pertinentes à gestão o farmacêutico é um profissional fundamental para compreensão dos pacientes e familiares sobre os princípios ativos medicamentosos e como a utilização racional pode ser benéfica em longo prazo.

Zanella, Aguiar Storpirtis (2015) demonstraram que medicamentos utilizados no tratamento de transtornos mentais possuem um impacto significativo ao paciente e que cabe ao farmacêutico, através da atenção farmacêutica promoção do sucesso terapêutico, pois, compreende a possibilidade de interações medicamentosas, acompanha o paciente do CAPS, avalia o processo de prescrição junto ao médico responsável pelo Centro e analisa se as interações prejudicam ou não o tratamento do paciente, assim, com a adoção de pedidas preventivas consegue evitar problemas relacionados aos medicamentos.

Carvalho et al., (2015) este estudo foi responsável por realizar um levantamento dos principais medicamentos usados em CAPS juvenil dentre eles as medicações risperidona, haloperidol, clorpromazina, fluoxetina e amitriptilina foram os principais medicamentos utilizados, os jovens entrevistados reconheciam a nomeclatura do medicamento, mas, não sabiam a sua classificação, ou seja, não tinham conhecimento de que por exemplo a fluoxetina é um espécie de antidepressivo e assim a 
assistência farmacêutica passou a realizar uma função orientativa, porque é muito importante que estes jovens saibam o porque estão utilizando este medicamento e quais os possíveis medicamentos têm a capacidade de interagir com outros medicamentos.

Leite et al., (2016) destacaram que no CAPS diversos níveis de transtornos mentais são enfrentados diariamente e que o farmacêutico deve compreender como cada medicamento poderá interagir com outros medicamentos, pois, no centro não ministrados antipsicóticos, antidepressivos, neurolépticos, antieplético e benzodiazepínicos e que via de regra os pacientes tendem a precisar de mais de uma medicação, sendo importante que o farmacêutico compreenda as possibilidades de interação medicamentosa e oriente não apenas o paciente, mas, também o médico responsável pela prescrição, pois, é o farmacêutico do CAPS o profissional que conhece quais medicamentos são de uso diário, contínuo e esporádico.

Almeida et al., (2016) trata sobre um estudo para desenvolvimento de um sistema de gestão autônoma de medicação, que é possível controlar quais medicamentos são ministrados a cada paciente registrado no sistema, isto permite que o farmacêutico avalie a utilização destes, dessa forma na pesquisa concluiu-se que profissionais de saúde e cuidadores podem fazer uso do guia e assim desenvolver uma autonomia dos pacientes porque isso os auxiliará a seguir corretamente o tratamento.

Silva e Lima (2017) demonstraram que houve uma evolução do papel do farmacêutico no CAPS, que deixou de ser meramente para cumprir uma obrigação sanitária e passou a contribuir efetivamente não apenas no tratamento do paciente, mas, também na gestão e distribuição dos fármacos, de modo que este compreende as quantidades e dosagens necessárias e como elas saem da farmácia e se realmente são utilizadas e benéficas para os pacientes do CAPS.

Scherer Berwing e Santiago (2017) a assistência farmacêutica cobre todo o processo de seleção, programação, aquisição, armazenamento e distribuição de medicamentos. Todas as etapas desse ciclo devem ser conduzidas pelo farmacêutico como profissional de referência, e o contato direto com o paciente ocorre durante o período de dispensação, pois, ele conseguirá apresentar e fazer com que o paciente compreenda o impacto desta medicação.

Santos (2018) demonstrou que a automedicação é um dos principais fatores que prejudicam o tratamento eficaz de pacientes com transtornos mentais isso porque a possibilidade de interação medicamentosa, ou ainda, agravamento do quadro é real, pois o paciente não tem consciência do perigo de tomar um medicamento não prescrito, nesse sentido é que se reforça o papel do farmacêutico enquanto gestor e orientador e que proporciona meios para adesão dos medicamentos psicotrópicos.

Smanio et al., (2018) demonstrou que combinar as atividades farmacêuticas com outras atividades multiprofissionais promovem efetivamente a saúde dos usuários e lhes proporcionam mais segurança e cumprimento das regulamentações. No entanto, embora esses avanços sejam claros, ainda faltam outros elementos para mensurar e documentar as atividades farmacêuticas desenvolvidas pela unidade para fortalecer a assistência medicamentosa em serviço.

Santos (2019) demonstrou a importância do farmacêutico no acompanhamento dos usuários de psicofármacos em dois CAPS no interior da Bahia de modo que de demonstrou o pouco conhecimento da equipe e dos usuários sobre a farmacologia terapêutica de modo que por não ter conhecimento dos medicamentos havia uma resistência dos pacientes ao tratamento, de modo que através da Assistência Farmacêutica foi possível reconhecer o tratamento e aderir a ele pois, juntos o paciente e os profissionais do CAPS desmitificaram o tratamento e os fármacos.

Beutinger e Limberger (2019) contataram que existe uma dificuldade de acesso aos medicamentos direcionados aos usuários do CAPi, isso porque a manutenção do centro requer investimento público e a demanda do CAPS analisado é alta, destacou-se também, que o farmacêutico é um profissional extremamente importante porque ele ajuda na distribuição racional dos medicamentos existentes na farmácia do centro e por compreender a relevância deste uso racional promove ao paciente qualidade de vida e melhora em seu tratamento. 
Guimarães (2020) trazem a assistência farmacêutica enquanto aspecto fundamental para o tratamento de pacientes com problemas correlacionados à saúde mental, e há uma necessidade de adequações físicas e pertinentes ao trabalho em equipe para que se consiga providenciar ao paciente um tratamento eficaz e adequado as suas condições.

Por fim, no estudo de Amaral et al., (2021) foram avaliados os critérios de identificação da saúde mental, o atendimento realizado na atenção básica e assistência farmacêutica voltada à saúde mental, este estudo analisou quatro cidades Campinas, Fortaleza, Porto Alegre e São Paulo e concluiu que a assistência à saúde mental sofre diversos problemas em decorrência da falta de medicamentos e de profissionais que de fato exercem uma assistência clara ao paciente, isso porque nas cidades de São Paulo e Campinas apenas as famílias tem conhecimento e contato com o farmacêutico, sendo necessário incluir o paciente também como protagonista do processo terapêutico.

\section{Limitações e viés}

Trata-se de uma pesquisa rigorosa que é usada para identificar e fornecer uma síntese da literatura mais recente sobre o uso da simulação da realidade como um intermediário no processo de ensino da formação farmacêutica. No entanto, devido aos diferentes sinônimos dos descritores principais, os artigos elegíveis podem ser perdidos. Existem alguns estudos em pequena escala com limitações e sem resultados confiáveis.

Os autores dos estudos selecionados alertaram para a cautela na interpretação dos resultados, principalmente considerando a dificuldade de agrupamento de dados e a heterogeneidade dos estudos em termos de mudanças, tipos, intensidade e diversidade de indicadores e o uso de síntese narrativa, pois eles não têm meta-análise.

\section{Conclusão}

A partir desta revisão foi possível compreender que o farmacêutico é um profissional essencial no tratamento realizado no CAPS porque ele exerce funções de gestão, assistência, informação, tecnicidade, de modo a viabilizar a eficácia do tratamento porque ensina aos pacientes, familiares e a própria equipe do CAPS como utilizar os medicamentos de forma adequada.

\section{Contribuições dos autores}

O conteúdo da revisão é de exclusiva responsabilidade individual dos autores.

\section{Declaração de conflito de interesses}

Os autores declaram que a pesquisa foi conduzida na ausência de quaisquer relações comerciais ou financeiras que possam ser interpretados como um potencial conflito de interesse.

\section{Referências}

Alencar, B. R. (2013). Processo de trabalho no Programa Saúde da Família: um enfoque na assistência farmacêutica. UEFS. 44(43):1-190. http://tede2.uefs.br:8080/bitstream/tede/179/2/BRUNO_ALENCAR_Disserta\%C3\%A7\%C3\%A3o_final.pdf

Almeida, H. C. M., Lopes, E. D., Gomes J. O., Silva, C. F., Lima, A. C., \& Gondim, A. P. (2016). Centro de assistência farmacêutica e saúde mental para o desenvolvimento de habilidades e competências. Encontros Universitários da UFC. 1(1): 3664-3770. http://repositorio.ufc.br/bitstream/riufc/56873/1/2016_resumo_eve_hcmalmeida.pdf

Amaral, C. E. M., Treichel, C. A. S., Francisco, P. M. S. B., \& Onocko-Campos, R. T.(2021). Assistência à saúde mental no Brasil: estudo multifacetado em quatro grandes cidades. Cadernos de Saúde Pública [online].37(3): 456-467. https://doi.org/10.1590/0102-311X00043420.

Beutinger, D., \& Limberger, J. B. (2019) Interfaces entre a assistência farmacêutica e o projeto terapêutico singular sob o olhar de profissionais de um CAPSi. Revista Eletrônica Disciplinarum Scientia. 20(2):239-256. https://periodicos.ufn.edu.br/index.php/disciplinarumS/article/view/2661/2396. 
Bezerra, I. C. (2013). Uso de psicofármacos na atenção psicossocial: sujeito, autonomia e corresponsabilização. Dissertação (Mestrado em Saúde Coletiva) Universidade Estadual do Ceará,56(44): 1-100.

Brasil. Ministério da Saúde. Portaria n 336, de 19 de fevereiro de 2002. Define e estabelece diretrizes para o funcionamento dos Centros de Atenção Psicossocial. Brasília, DF: Ministério da Saúde; 2002a.

Brasil. Ministério da Saúde. Portaria n 189, de 20 de março de 2002. Brasília, DF: Ministério da Saúde; 2002 b.

Carvalho, I. L. N., Gondim, A. P. S., Pena, P. F. A.; Moreira, G. A. R.; \& Feitosa, , T. H. P. (2015). Perfil de tratamento medicamentoso dos adolescentes atendidos nos Centros de Atenção Psicossocial Infanto-Juvenil do Município de Fortaleza - CE. Anais do 11 ${ }^{\circ}$ Congresso Brasileiro de Saúde Coletiva, 67(65): 244-264.

Correia, G. A. R., \& Gondim, A. P. S. (2014) Utilização de benzodiazepínicos e estratégias farmacêuticas em saúde mental. Revista Saúde Debate. 38(201):393-398. https://doi.org/10.5935/0103-1104.20140036.

Coutinho, M. B. Atuação farmacêutica no campo de saúde mental: uma revisão da literatura. UFPB - Universidade Federal da Paraíba. João Pessoa - PB, 2015 .

Cunha, P. L. P., Cunha, C. S., \& Alves, P. F. (2014) Revisão bibliográfica sistemática integrativa: a pesquisa baseada em evidências. Revista Anima Educação. 6(3):1-63. http://dx.doi.org/10.1590/ S0103-21002007000200001.

Guimarães, S. S. (2020). Assistência farmacêutica no contexto da atenção primária à saúde de um município brasileiro. Universidade Federal do Ceará. Repositório Institucional: 1-112. http://www.repositorio.ufc.br/handle/riufc/57366.

Leite, L. O. B., Salgado, P. R. R., Rosa, S. P. S., Gonçalves, S. A. A.; Medeiros, A. P., Dias, J. M. F., \& Paiva, A. C. C. (2016) Os principais medicamentos prescritos em centros de apoio psicossocial - CAPs. Informativo Técnico do Semiárido. 10(2): 76-91. http://www.gvaa.com.br/revista/index.php/INTESA.

Santos, A. M. (2018). A atuação do farmacêutico na saúde mental após a reforma psiquiátrica: uma revisão de literatura. Universidade Federal de Uberlândia. 77(76): 1-24. https://repositorio.ufu.br/bitstream/123456789/22923.pdf.

Santos, B. J. R. (2019). A importância do profissional farmacêutico no acompanhamento aos usuários de psicofármacos em dois CAPS no interior na Bahia. Faculdade Maria Milza. 18(24): 1-57. http://131.0.244.66:8082/jspui/bitstream/123456789/1624/2/TCC\%20II.pdf

Scherer Berwing, N. D., \& Santiago, E. S. (2017). Profissional farmacêutico nos centro de atenção psicossocial. UFMT- ${ }^{\circ}$ jornada regional de saúde mental teles pires. 44(3): 435-470.

Silva, S. H. (2014). Avaliação da assistência farmacêutica nos Centros de Atenção Psicossocial da Região do Médio Paraopeba - MG. Universidade Federal de Minas Gerais. 45(45): 1-143. https://repositorio.ufmg.br/bitstream/1843/BUOS-AT6LJU/1/disserta_o_sarah_vers_o_final_impressao__2_.pdf.

Silva, S. N., \& Lima, M. G (2017). Assistência Farmacêutica na Saúde Mental: um diagnóstico dos Centros de Atenção Psicossocial. Ciência \& Saúde Coletiva [online]. 22(6): 2025-2036. https://doi.org/10.1590/1413-81232017226.25722016.

Smanio, D. V., Silva, E. M., Santos, M. M. H., \& Guimarães, F. H. L. (2018). Atividades desenvolvidas pela assistência farmacêutica no CAPS-AD Estação Vicente Araújo localizado na cidade de Recife-PE. Faculdade Pernambucana de Saúde. Repositório Institucional: 1-59.. http://tcc.fps.edu.br:80/jspui/handle/fpsrepo/266

Zanella, C. G., Aguiar, P. M., \& Storpirtis, S (2015). Atuação do farmacêutico na dispensação de medicamentos em Centros de Atenção Psicossocial Adulto no município de São Paulo, SP, Brasil. Ciência \& Saúde Coletiva, 20(2): 325-332. https://doi.org/10.1590/1413-81232015202.17872013. 